\title{
Peregrine Soliton and Akhmediev Breathers in a Chameleon Electrical Transmission Line
}

\author{
Bedel Giscard Onana Essama ${ }^{1,2^{*}}$, Salome Ndjakomo Essiane1, Frederic Biya-Motto ${ }^{2}$, \\ Bibiane Mireille Ndi Nnanga ${ }^{3}$, Mohammed Shabat ${ }^{4}$, Jacques Atangana ${ }^{5}$
}

\author{
${ }^{1}$ Applied Biotechnology and Engineering Laboratory, Department of Electrical Engineering, Higher Technical Teachers, Training \\ College (HTTTC) of EBOLOWA, University of Yaounde I, Yaounde, Cameroon \\ ${ }^{2}$ Laboratory of Electronics, Department of Physics, Faculty of Science, University of Yaounde I, Yaounde, Cameroon \\ ${ }^{3}$ Laboratory of Mechanics, Department of Physics, Faculty of Science, University of Yaounde I, Yaounde, Cameroon \\ ${ }^{4}$ Department of Physics, Islamic University of Gaza, Gaza, Palestine \\ ${ }^{5}$ Higher Teacher Training College of Yaounde, University of Yaounde I, Yaounde, Cameroon \\ Email: *onanaessama@yahoo.fr
}

How to cite this paper: Essama, B.G.O., Essiane, S.N., Biya-Motto, F., Nnanga, B.M.N., Shabat, M. and Atangana, J. (2020) Peregrine Soliton and Akhmediev Breathers in a Chameleon Electrical Transmission Line. Journal of Applied Mathematics and Physics, 8, 2775-2792.

https://doi.org/10.4236/jamp.2020.812205

Received: April 29, 2020

Accepted: December 4, 2020

Published: December 7, 2020

Copyright $\odot 2020$ by author(s) and Scientific Research Publishing Inc. This work is licensed under the Creative Commons Attribution International License (CC BY 4.0).

http://creativecommons.org/licenses/by/4.0/

\begin{abstract}
We analyze the particular behavior exhibited by a chaotic waves field containing Peregrine soliton and Akhmediev breathers. This behavior can be assimilated to a tree with "roots of propagation" which propagate randomly. Besides, this strange phenomenon can be called "tree structures". So, we present the collapse of dark and bright solitons in order to build up the above mentioned chaotic waves field. The investigation is done in a particular nonlinear transmission line called chameleon nonlinear transmission line. Thus, we show that this line acts as a bandpass filter at low frequencies and the impact of distance, frequency and dimensionless capacitor are also presented. In addition, the chameleon's behavior is due to the fact that without modifying the appearance structure, it can present alternatively purely right- or left-handed transmission line. This line is different to the composite one.
\end{abstract}

\section{Keywords}

Freak Waves Generation, Transmission Line, Metamaterials, Right/Left-Handed Behaviors, Tree Structures, Collective Coordinates

\section{Introduction}

Metamaterials are materials which have both the permeability $(\mu)$ and the permittivity $(\varepsilon)$ parameters are set negative at the same frequency [1] [2]. This kind of materials is often called double-negative material or left-handed metamaterials [1] [2]. This class of materials has negative refractive index. So, they 
are not found in nature but they can be artificially designed [2]. At microwave frequencies transmission lines have been proposed [3], a special left-handed transmission line [4], and composite right/left-handed transmission line [5].

The analysis of extreme wave events has formed one of the most useful points of intense theoretical investigations and physical applications [6] [7]. Several researches and diverse experiments have been done in order to observe the emergence of extreme events in many physical domains. Among them, we have nonlinear optics [8], modelocked lasers [9], hydrodynamics [10], and plasmas [11]. The most known rogue events are Peregrine solitons [12], Kuznetsov-Ma breathers [13] [14], and Akhmediev breathers [15].

One of the most significant rogue waves mechanism of generation is modulation instability which can be seen as a fundamental property of many nonlinear dispersive systems [16]. This phenomenon is associated with the growth of periodic perturbations on a continuous-wave background [16]. In optics, modulation instability comes from noise results in a series of high-contrast peaks of random intensity [17] [18]. These localized peaks are compared to ocean rogue waves [19]. More so, significant researches on modulation instability are been done in some domains such as nonlinear optics [20] [21], hydrodynamics [22], and electrical transmission lines [23].

However, there exists a strange phenomenon directly related to the investigation of extreme events. It appears when a chaotic waves field is generated by modulation instability. This expanding structure corresponds to the so-called "tree structure" [24]. Some investigations have been done in literature concerning this phenomenon. Among them, there is the "Christmas tree" which appears during the formation of Peregrine waves [25]. Such phenomenology has been firstly observed and extensively analyzed in [26], which is related to the umbilical gradient catastrophe. It has been also shown that Raman effect can induce the appearance of particular "tree structure" with roots which can be called "roots of propagation" [24] [27]. Authors else such as Dudley [28], sustains that such "tree structures" correspond to signatures of analytic nonlinear Schrödinger equation solutions in chaotic modulation instability [24]. Furthermore, to the best of our knowledge, the characterization of electromagnetic wave behavior, the description of internal excitation leading to specific rogue waves generation, the so-called tree structures corresponding to rogue events signature in a chameleon transmission line, have been least reported.

In this paper, based on the work of Fukushima et al. [29], Marquié et al. [23] and that of Togueu et al. [30], we model the electromagnetic wave behavior by a nonlinear Schrödinger equation. Thus, this equation includes second-order dispersion and cubic-nonlinearity in a modulable transmission line. The coefficients of this equation are expressed as function of frequency. This electromagnetic wave is analyzed when right-handed behavior and left-handed one alternatively exhibited in the line. The chameleon's behavior of this particular line is verified. Furthermore, some specific conditions allowing the line to act as a filter are presented at low frequencies. More so, we also present internal and physical 
conditions leading to special rogue events such as the so-called tree structures. We demonstrate that the line can support dark and bright soliton solutions. Moreover, collective coordinates technique and the Gaussian ansatz function are used [31], in order to characterize the light pulse.

The paper is organized as follows. In Section 2, we present the logarithmic nonlinearity for the capacitance, the voltage propagation equations and the nonlinear Schrödinger equation model. The coefficients of this last equation are plotted in order to improve the comprehension of strange phenomena studied. Thereafter, we apply the collective coordinates technique in order to obtain the collective coordinate equations of motion. In Section 3, we apply numerical experiments in order to investigate numerically the collective coordinates and present the results. The outcomes are summarized in Section 4.

\section{Mathematical Description of the Model}

\subsection{Preliminaries}

The model under consideration represents a modulable nonlinear electrical transmission line where elementary cell is illustrated in Figure 1 [29] [30].

Each unit cell, such as the $n^{\text {th }}$ one, contains a linear inductor $L_{1}$ in parallel with a linear capacitor $C_{1}$ in the series branch and a linear inductor $L_{2}$ in parallel with a nonlinear capacitor $C\left(V_{n}\right)$ in the shunt branch. Here we assume that the logarithmic nonlinearity for the capacitance is given by [32] [33]:

$$
C\left(V_{n}\right)=\frac{V_{0} C_{0}}{V_{n}} \ln \left(1+\frac{V_{n}}{V_{0}}\right)
$$

where $V_{0}$ and $C_{0}$ take constant values.

Applying Kirchhoff's laws to the circuit model, we can obtain the following voltage propagation equations [30]:

$$
\begin{aligned}
& \left(V_{0}+V_{n}\right) \frac{\mathrm{d}^{2} V_{n}}{\mathrm{~d} t^{2}}-\left(\frac{\mathrm{d} V_{n}}{\mathrm{~d} t}\right)^{2} \\
& =\frac{\left(V_{0}+V_{n}\right)^{2}}{V_{0}}\left\{\left(u_{0}^{2}+C_{r} \frac{\mathrm{d}^{2}}{\mathrm{~d} t^{2}}\right)\left(V_{n+1}+V_{n-1}-2 V_{n}\right)-\omega_{0}^{2} V_{n}\right\}
\end{aligned}
$$

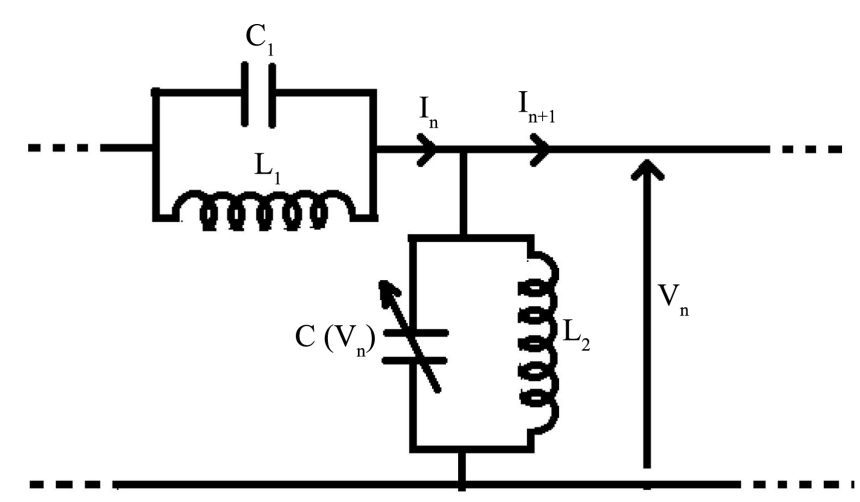

Figure 1. Model for the unit-cell circuit. 
where $C_{r}=\frac{C_{1}}{C_{0}}, u_{0}^{2}=\frac{1}{L_{1} C_{0}}$ and $\omega_{0}^{2}=\frac{1}{L_{2} C_{0}}$. Equation (2) shows that an additional dispersion coefficient $C_{r}$ is considered on the line. Thereafter, the numerical simulations will consider the following parameters [23] [30]: $L_{1}=680 \mu \mathrm{H}$, $L_{2}=470 \mu \mathrm{H}, C_{0}=470 \mathrm{pF}$ and $V_{0}=3.9 \mathrm{~V}$.

The capacitor $C_{1}$ is considered as a free parameter with physically acceptance value [30]. According to the investigations done in [30], the parameter $C_{r}$ will impose the behavior of the transmission line. So, they show that when $C_{r}<\frac{L_{2}}{L_{1}}$ we have the right-handed behavior, but for $C_{r}>\frac{L_{2}}{L_{1}}$, the transmission line exhibits left-handed behavior. It is important to note that a rapid calculation leads to $\frac{L_{2}}{L_{1}} \approx 0.691$. This situation justifies the name "Chameleon transmission line" because the line changes its behavior but does not modify its external aspect.

\subsection{Theoretical Model for Electrical Transmission Line}

The nonlinear Schrödinger equation inspired from [30], but reformulated in terms of slowly varying envelope of the electric field $A(Z, \tau)$ as follows [34]:

$$
\frac{\partial A}{\partial Z}=-i \frac{\Theta_{2}}{2} \frac{\partial^{2} A}{\partial \tau^{2}}+i \Upsilon|A|^{2} A
$$

where $A(Z, \tau)$ is the slowly varying envelope of the electric field at position $Z=\varepsilon\left(n-V_{g} t\right)$ and at time $\tau=\varepsilon^{2} t$ [30]. Here $\varepsilon$ is a positive and small parameter. The terms $\Theta_{2}$ and $\Upsilon$ are second-order dispersion and cubic-nonlinearity, respectively [35] [36] [37] [38]. These above mentioned coefficients are defined as follows [30]:

$$
\begin{gathered}
\Theta_{2}=-\frac{V_{g}^{2}}{2 \omega}+\frac{\left(u_{0}^{2}-C_{r} \omega^{2}\right) \cos (k)-4 C_{r} V_{g} \omega \sin (k)}{2 \omega\left[1+4 C_{r} \sin ^{2}\left(\frac{k}{2}\right)\right]} \\
\Upsilon=\frac{3 \omega}{2 V_{0}^{2}}\left[-1+\frac{\left.8 C_{r} \sin ^{2}\left(\frac{k}{2}\right)\right]}{\left.1+4 C_{r} \sin ^{2}\left(\frac{k}{2}\right)\right] .}\right.
\end{gathered}
$$

where the wave number $k$ is taken in the Brillouin zone. This dispersion relation admits two cutoff frequencies at $k=0 \mathrm{rad} \cdot \mathrm{Cell}^{-1}$ and $k=\pi \mathrm{rad} \cdot \mathrm{Cell}^{-1}$ [30]. The group velocity is given by [30]:

$$
V_{g}=\frac{\left(u_{0}^{2}-C_{r} \omega_{0}^{2}\right) \sin (k)}{\omega\left[1+4 C_{r} \sin \left(\frac{k}{2}\right)^{2}\right]^{2}} .
$$

The group velocity $V_{g}$ plays a key role in the nature of waves.

\subsection{Collective Coordinate's Theory}

The collective coordinate's technique is a great method of characterization of a 
light pulse intensity profile using ansatz functions [39] [40]. The variational equations are essential to obtain a good description of the light pulse [35] [36].

\subsubsection{Conventional Gaussian Ansatz Function}

For our variational analysis, the desired form of the Gaussian ansatz function $f$ is given by [37] [38]:

$$
\begin{aligned}
f(Z, \tau)= & X_{1}(Z) \exp \left[-\left(\frac{\tau-X_{2}(Z)}{X_{3}(Z)}\right)^{2}+\frac{1}{2} i X_{4}(Z)\left(\tau-X_{2}(Z)\right)^{2}\right. \\
& \left.+i X_{5}(Z)\left(\tau-X_{2}(Z)\right)+i X_{6}(Z)\right]
\end{aligned}
$$

where $X_{1}, X_{2}, \sqrt{2 \log 2} X_{3}, \frac{X_{4}}{2 \pi}, X_{5}, X_{6}$ are the conventional collective coordinates often used to represent the pulse amplitude, temporal position, full width at half-maximum (FWHM) of peak power, chirp, frequency shift and phase, respectively [41] [42].

\subsubsection{Collective Coordinate Equations of Motion.}

Then, the equations of motion obtained from Bare approximation [39] [40], are given by the following expressions [31] [42]:

$$
\begin{gathered}
\dot{X}_{1}(Z)=\frac{1}{2} X_{1} X_{4} \Theta_{2}(Z) \\
\dot{X}_{2}(Z)=-X_{5} \Theta_{2}(Z) \\
\dot{X}_{3}(Z)=-X_{3} X_{4} \Theta_{2}(Z) \\
\dot{X}_{4}(Z)=\left(X_{4}^{2}-\frac{4}{X_{3}^{4}}\right) \Theta_{2}(Z)-\sqrt{2} \frac{X_{1}^{2}}{X_{3}^{2}} \Upsilon(Z) \\
\dot{X}_{5}(Z)=0 \\
\dot{X}_{6}(Z)=\left(\frac{1}{X_{3}^{2}}-\frac{1}{2} X_{5}^{2}\right) \Theta_{2}(Z)+\frac{5 \sqrt{2}}{8} X_{1}^{2} \Upsilon(Z) .
\end{gathered}
$$

Equations (8)-(13) present the variational equations which are function of the coefficients $\Theta_{2}(Z)$ and $\Upsilon(Z)$. All these equations are influenced by $\Theta_{2}(Z)$ except the dynamics of frequency shift, $\dot{X}_{5}(Z)$, which is only closed to zero. Furthermore, the quantity $\Upsilon(Z)$ is only acted on $\dot{X}_{4}(Z)$ and $\dot{X}_{6}(Z)$. However, the most important point is that all the variational equations are strongly dependant on frequency $\omega$ [37] [38].

\section{Numerical Experiments}

\subsection{Initial Conditions at the Beginning of the Propagation}

The initial conditions at the beginning of the propagation are the same compared to those used in [35] [36] [37] [38]. For the analysis of our optical system the wave number is taken as $k=3 \mathrm{rad} \cdot \mathrm{Cell}^{-1}$. Otherwise, two lengths of propagation will be taken such as $Z=3 \times 10^{-24} \mathrm{~m}$ and $Z=10^{-6} \mathrm{~m}$. Figures 2-4 show 


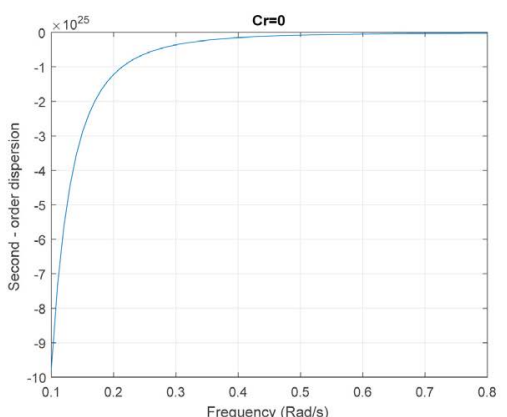

(a)

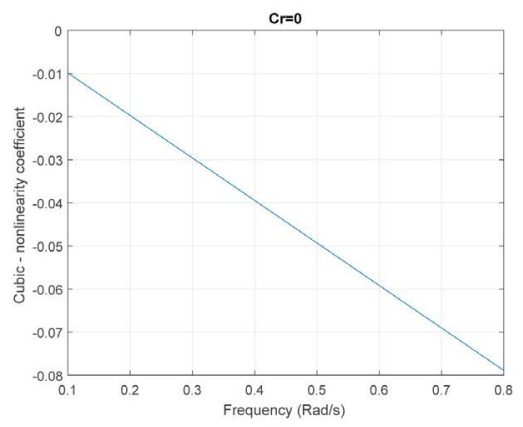

(c)

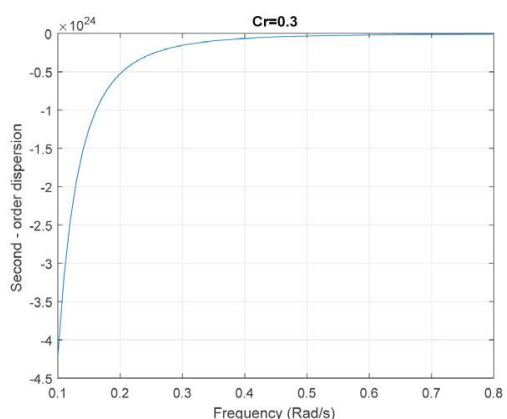

(b)

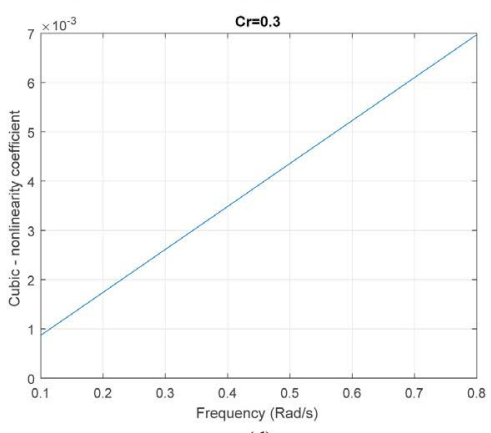

(d)

Figure 2. Frequency dependencies of the coefficients of Equation (3). (a) Second-order dispersion: (a) $C_{r}=0$ and (b) $C_{r}=0.3$. Cubic-nonlinearity: (c) $C_{r}=0$ and (d) $C_{r}=0.3$.

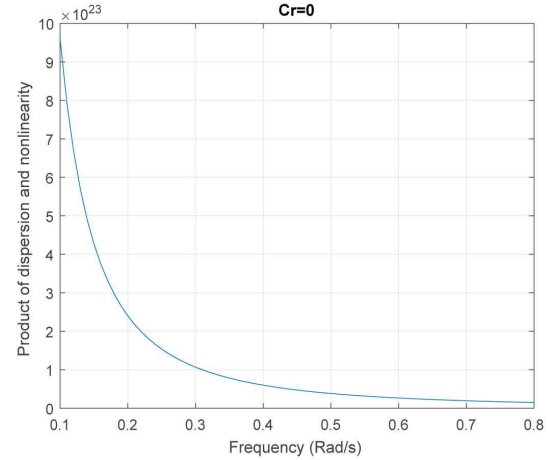

(a)

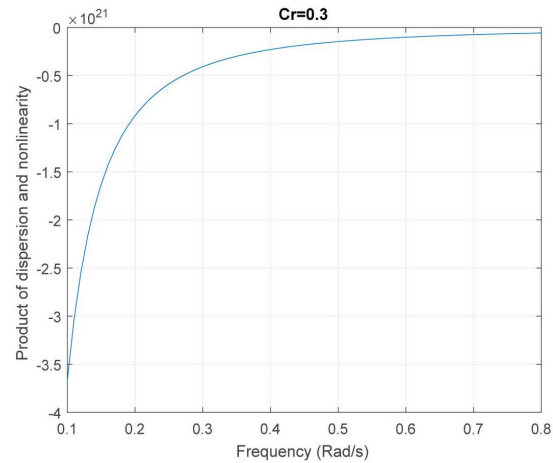

(b)

Figure 3. Frequency dependencies of the coefficients of Equation (3). Product of dispersion and nonlinearity (a) $C_{r}=0$ and (b) $C_{r}=0.3$.

frequency dependencies of coefficients of Equation (3) for $C_{r}=0, C_{r}=0.3$, $C_{r}=1, C_{r}=1.5$ and $C_{r}=10$. These figures present the variations of second-order dispersion and that of cubic-nonlinearity. In addition, the variations of the product of these two effects are also included. According to the collective coordinate representation, the solid red curve corresponds to the dynamics of collective coordinates obtained from bare approximation (Gaussian Ansatz function) as depicted in Figure 5(a) and Figure 5(b). Further, the dashed black curves represent the collective coordinates coming from minimization. Thus, the dotted green curve gives the residual field energy (RFE) [35] [36] [37] [38]. 


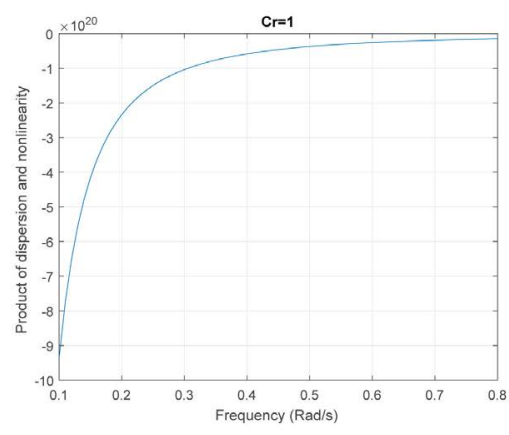

(a)

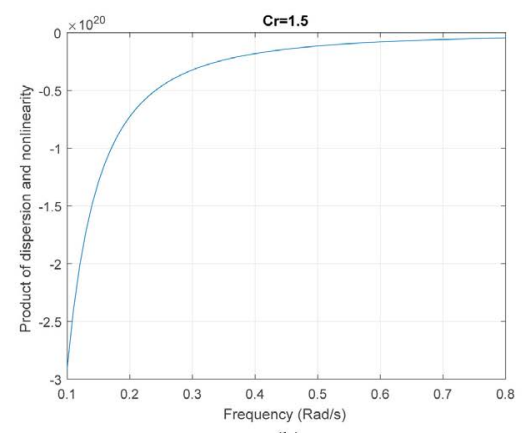

(b)

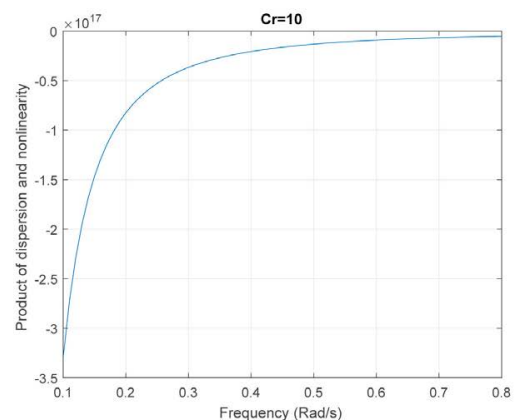

(c)

Figure 4. Frequency dependencies of the product of dispersion and nonlinearity coefficients for Equation (3): (a) $C_{r}=1$. (b) $C_{r}=1.5$; (c) $C_{r}=10$.
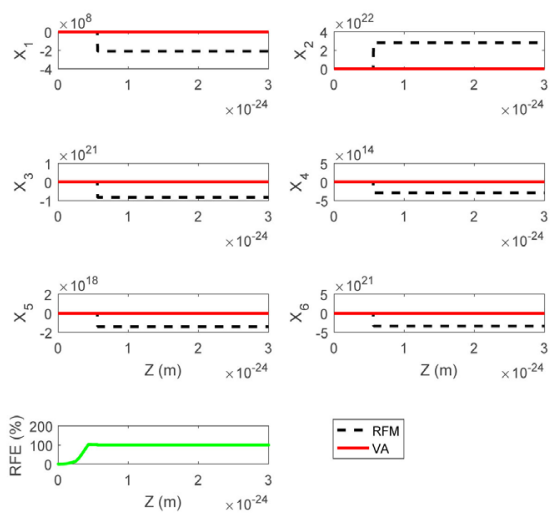

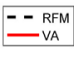

(a)
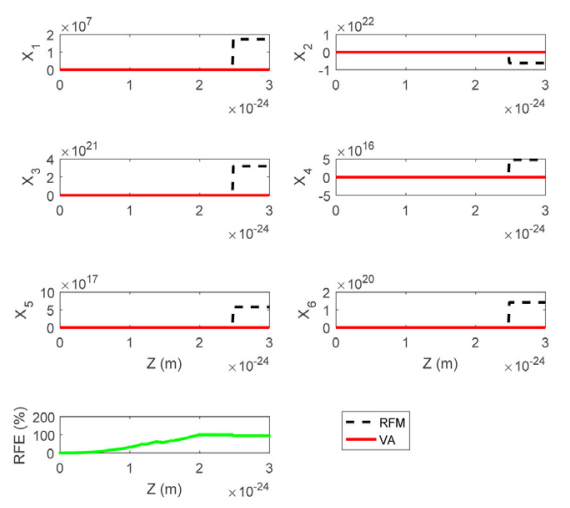

\begin{tabular}{l}
$-{ }_{\text {RFM }}$ \\
\hline
\end{tabular}

(b)

Figure 5. Chaotic wave's field for $C_{r}=0$. Dynamic of collective coordinates: (a) $\omega=0.25 \mathrm{rad} / \mathrm{s}$; (b) $\omega=0.35 \mathrm{rad} / \mathrm{s}$ [Frequency ranges considered: $0 \leq \omega \leq 0.78 \mathrm{rad} / \mathrm{s}$ and $\omega>0.78 \mathrm{rad} / \mathrm{s}$ at $Z=3 \times 10^{-24} \mathrm{~m} \mathrm{]}$.

Figure 6(a) and Figure 6(b) represent full numerical equations [35] [36] [37]. Moreover, Figure 6(c) and Figure 6(d) show 2D full numerical equations [24] [27] [28].

\subsection{Introduction of Right-Handed Propagation}

The right-handed behavior occurs on the line when $0 \leq C_{r}<0.691$ [30]. Consequently, two cases will be investigated known as $C_{r}=0$ and $C_{r}=0.3$. 


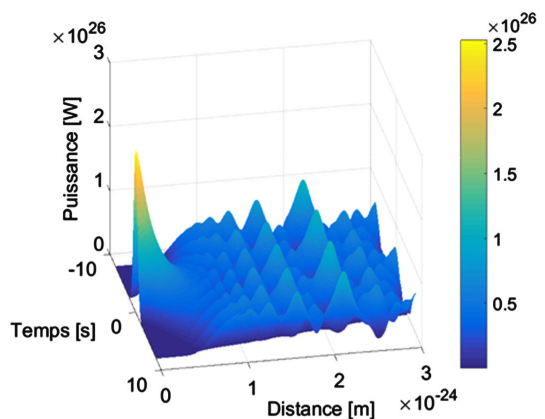

(a)

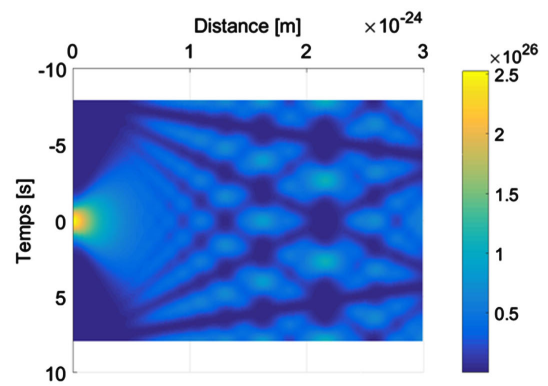

(c)

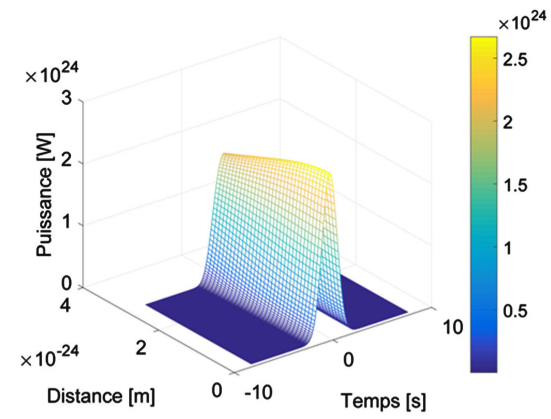

(b)

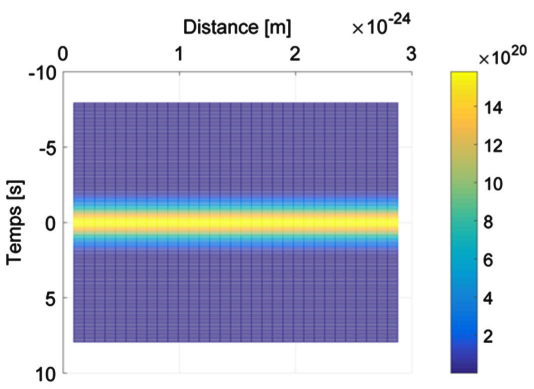

(d)

Figure 6. Chaotic wave's field for $C_{r}=0$. Full equations: (a) $\omega=0.25 \mathrm{rad} / \mathrm{s}$; (b) $\omega=0.78 \mathrm{rad} / \mathrm{s}$. 2D Full equations: (c) $\omega=0.25 \mathrm{rad} / \mathrm{s}$; (d) $\omega=5 \mathrm{rad} / \mathrm{s}$ [Frequency ranges considered: $0 \leq \omega \leq 0.78 \mathrm{rad} / \mathrm{s}$ and $\omega>0.78 \mathrm{rad} / \mathrm{s}$ at $Z=3 \times 10^{-24} \mathrm{~m} \mathrm{]}$.

\subsubsection{Akhmediev-Peregrine Waves Field Generation at Low Frequencies}

The first considered case is $C_{r}=0$ at low frequencies $0<\omega<0.78 \mathrm{rad} / \mathrm{s}$ for $Z=3 \times 10^{-24} \mathrm{~m}$. When the frequency is given such as $\omega=0.25 \mathrm{rad} / \mathrm{s}$ we obtain $\Theta_{2}=-0.5 \times 10^{25} \mathrm{ps}^{2} \cdot \mathrm{m}^{-1}$ and $\Upsilon=-0.025 \mathrm{~W}^{-1} \cdot \mathrm{m}^{-1}$ as depicted in Figure 2(a) and Figure 2(c). Besides, the product $\Theta_{2} \Upsilon>0$, so the nonlinear Schrödinger model supports bright soliton solution as seen in Figure 3(a). The strong negative nonlinearity acts on the weak negative dispersion in order to provoke the multiplication of several broken points presented in Figure 5(a). In fact, all the dynamic of collective coordinates coming from minimization are broken at $Z=0.5 \times 10^{-24} \mathrm{~m}$. This situation reveals that a strange phenomenon acts in the system. So, the bright soliton has lost its stability. Besides, the residual field energy increases and approaches 100 percent. This information suggests an increase of internal distortions as depicted in Figure 5(a). The strong negative nonlinearity continues to act on the weak negative dispersion in order to induce the fragmentation of the bright soliton into several grains of activity [43]. Hence, once grains activated, the spatial inhomogeneity acts as a nonlocal coupling that provides a coherent build-up of an extreme event. These monster events continue to undergo distortions originating from the strong negative nonlinearity. Besides, this trouble situation provokes the multiplication of strong harmonic waves with several residual wave motions at adjacent sideband frequencies [16] [22] [43]. This situation induces the modulation of residual waves. Hence, this 
perturbed situation provokes the generation of a chaotic waves field containing one Akhmediev-Peregrine soliton [44] (left) and several Akhmediev breathers (right) [15] [43], as depicted in Figure 6(a).

\subsubsection{Tree Like-Structures at Low Frequencies}

The observation of $2 \mathrm{D}$ full equation of this perturbed waves field shows the fragmentation of the bright soliton as depicted in Figure 6(c). Besides, the collapse of this wave leads to a strange phenomenon. In fact, we observe a strange structure similar to a tree with the multiple roots [25]. Those roots propagate randomly in the optical system as seen in Figure 6(c). Then, this structure can be assimilated to a tree with many roots which can be called roots of propagation [25]. Besides, the most colored point in the left part corresponds to the Akhmediev-Peregrine wave. In the second part, the black big points stand for the Akhmediev waves train in which the amplitude waves reach $0.75 \times 10^{26} \mathrm{~W}$ as illustrated in Figure 6(c). The average black points also correspond to the average Akhmediev waves train. Their amplitudes approach $0.5 \times 10^{26} \mathrm{~W}$. Moreover, the very small black point stands for the most little Akhmediev waves train in which the waves amplitude reaches $0.25 \times 10^{26} \mathrm{~W}$ as depicted in Figure 6(c) and Figure $6(\mathrm{e})$. Further, similar rogue signatures have been studied. Among them we have the researches concerning specific tree structures called Christmas tree [25]. Consequently, this expanding structure, which is called Christmas tree appears to emerge past the formation of the original Peregrine peak [25]. As the structure expands, progressively at the peak emergence times more localized peaks arise. Furthermore, this strange tree structure has been also investigated in Kundu-Eckhaus equation by Bayindir [27]. Indeed, it has been assimilated to a chaotic waves field induced by modulation instability [27]. Otherwise, a similar situation has also been presented in optical field by Dudley et al. [28]. In fact, tree structure is assimilated to signatures of analytic nonlinear schrödinger equation solutions in chaotic modulation instability. Hence, the structure obtained is similar to a density map [28]. Then, according to previous investigations [25] [27], each rogue event has a particular signature corresponding to a specific tree structure. So, we present here the particular signature of a chaotic waves field containing Akhmediev-Peregrine waves, Peregrine waves and Akhmediev wave trains.

\subsubsection{Soliton Stability at High Frequencies}

We consider $C_{r}=0$ and the propagation at high frequencies is obtained for $\omega>0.78 \mathrm{rad} / \mathrm{s}$ for $Z=3 \times 10^{-24} \mathrm{~m}$. When the frequency increases from

$\omega=0.25 \mathrm{rad} / \mathrm{s}$ to $\omega=0.35 \mathrm{rad} / \mathrm{s}$, the strong negative nonlinearity continues to acts on the weak negative dispersion. This situation leads to the broken points exhibited by the dynamic of collective coordinates originating from minimization as illustrated in Figure 5(b). Indeed, this dynamics of collective coordinates begins to beak at $Z=2.5 \times 10^{-24} \mathrm{~m}$. This information denotes that perturbations least act in the system since they only influence the end of the propagation as 
depicted in Figure 5(b). If the frequency continues to increase from $\omega=0.35 \mathrm{rad} / \mathrm{s}$ to $\omega=0.78 \mathrm{rad} / \mathrm{s}$, the bright soliton gains in stability as depicted in Figure 6(b) where the robustness of the pulse is presented. In fact, the strong negative nonlinearity and the weak negative dispersion are completely compensated [24], and have built up a robust wave. Despite the action of perturbations depicted in Figure 5(b), the stability of bright soliton is totally restored at high frequency as shown in Figure 6(d) by the signature of the stable soliton. Moreover, similar soliton light pulse stability was recently investigated in ultracold bosonic seas by Charalampidis [25].

\subsection{Second Case of Right-Handed Propagation}

The second case of right-handed behavior of the electrical line is considered for $C_{r}=0.3$ [30]. When the frequency is considered such as $\omega=0.15 \mathrm{rad} / \mathrm{s}$ the second-order dispersion coefficient has a weak negative value $-10^{24} \mathrm{ps}^{2} \cdot \mathrm{m}^{-1}$ and the cubic-nonlinearity presents a strong positive value $1.5 \times 10^{-3} \mathrm{~W}^{-1} \cdot \mathrm{m}^{-1}$ as depicted in Figure 2(b) and Figure 2(d). The product of these two effects is $\Theta_{2} \Upsilon<0$ as illustrated in Figure 3(b). Hence, at low frequencies the dark soliton propagates. The soliton light pulse regains its stability as seen in Figure 7(a). Besides, it appears that the weak negative value of dispersion is completely compensated the strong positive value of the nonlinearity in order to build-up the dark soliton depicted in Figure 7 (a). This stability is maintained at high frequencies as illustrated in Figure 7 (b) for $\omega=0.15 \mathrm{Mrad} / \mathrm{s}$. It clearly appears that the introduction of $C_{r}=0.3$ modifies the behavior of the line at low frequencies. In fact, the line acts as a bandpass filter [30], which totally cleans and cancels the distortions acting at low frequencies as depicted in Figure 7.

\subsection{Introduction of Left-Handed Propagation}

The left-handed behavior occurs when $C_{r}>0.691$. Three cases will be investigated corresponding to $C_{r}=1, C_{r}=1.5$ and $C_{r}=10$. We consider the first mentioned case of left-handed behavior known as $C_{r}=1$. Figure 4 (a) presents $\Theta_{2} \Upsilon<0$

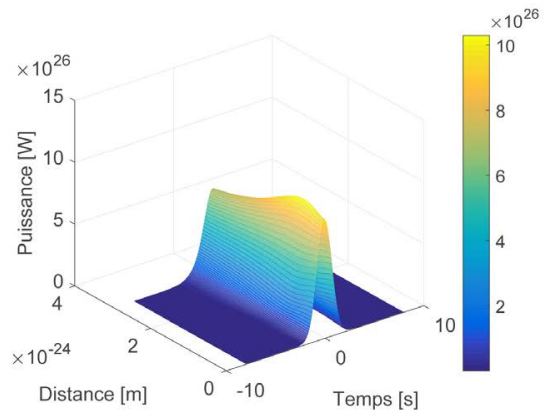

(a)

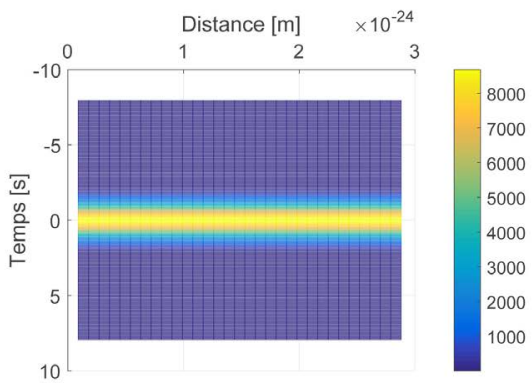

(b)

Figure 7. Soliton light pulse propagation for $C_{r}=0.3$. (a) Full equations $\omega=0.15 \mathrm{rad} / \mathrm{s}$; (b) $2 \mathrm{D}$ Full equations $\omega=0.15 \mathrm{Mrad} / \mathrm{s}$ [Frequency ranges considered: $0 \leq \omega \leq 0.78 \mathrm{rad} / \mathrm{s}$ and $\omega>0.78 \mathrm{rad} / \mathrm{s}$ at $\left.Z=3 \times 10^{-24} \mathrm{~m}\right]$. 
justifying the propagation of dark soliton. The dynamic of collective coordinates originating from Gaussian Ansatz practically reconstruct the behavior exhibited by that coming from minimization as depicted in Figure 8(a). Despite some disparities exhibited by those two dynamic, residual field energy approaches 0.02 percent. This fact suggests that the reconstruction is good as depicted in Figure 8(a) revealing the robustness of the soliton light pulse. This stability and this robustness are clearly maintained at low frequencies when the frequency increases from $\omega=0.15 \mathrm{rad} / \mathrm{s}$ to $\omega=0.25 \mathrm{rad} / \mathrm{s}$ as depicted in Figure 8(a) and Figure 9(a). In addition, this stable behavior exhibits its signature as illustrated in Figure 9(c).

Moreover, we consider the significant increase of the length of propagation from $Z=3 \times 10^{-24} \mathrm{~m}$ to $Z=10^{-6} \mathrm{~m}$. Besides, at high frequencies for $\omega=0.25 \mathrm{Mrad} / \mathrm{s}$ the dynamics of collective coordinates originating from minimization reconstructs that coming from Gaussian Ansatz as seen in Figure 8(b). The residual field energy confirms the good reconstruction since it reaches 0.2 percent. When the frequency significantly increases from $\omega=0.25 \mathrm{Mrad} / \mathrm{s}$ to $\omega=25 \mathrm{Mrad} / \mathrm{s}$, second-order dispersion and cubic-nonlinearity interact. This situation induces modulational instability leading to the collapse of the dark soliton. This above mentioned modulational instability provokes the coherent build-up of the chaotic waves field depicted in Figure 9(b). Indeed, as previously observed the chaotic waves field presents Akhmediev-Peregrine freak wave and Akhmediev waves trains as depicted in Figure 9(b). The signature of this chaotic waves field is also illustrated in Figure 9(d). However, the aspect of this chaotic waves field is practically identical to that obtained at low frequencies when right-handed propagation was considered as depicted in Figure $9(\mathrm{~d})$ and Figure $6(\mathrm{c})$. So, the significant increase of the length of propagation from $3 \times 10^{-24} \mathrm{~m}$ to $10^{-6} \mathrm{~m}$ induces a considerable decrease of soliton peak power from $2.5 \times 10^{26} \mathrm{~W}$ to $7 \mathrm{~W}$.

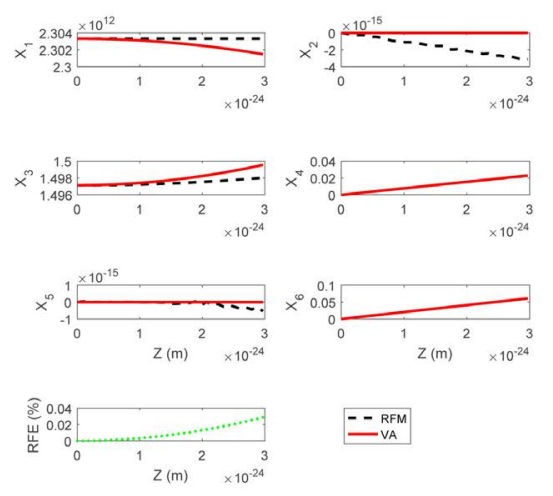

(a)

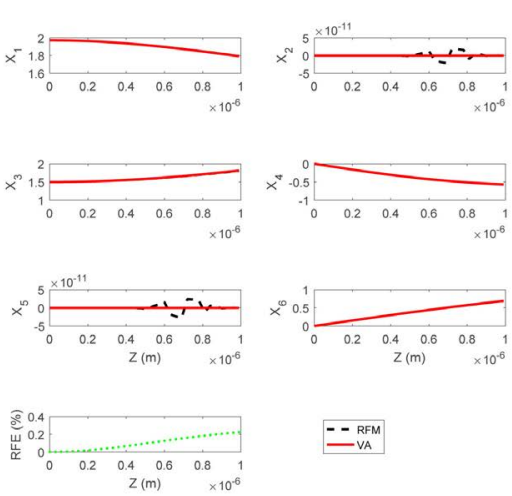

(b)

Figure 8. First case of left-handed behavior. Soliton light pulse and chaotic wave's field for $C_{r}=1$. Dynamic of collective coordinates: (a) $\omega=0.15 \mathrm{rad} / \mathrm{s}$ at $Z=3 \times 10^{-24} \mathrm{~m}$; (b) $\omega=0.25 \mathrm{Mrad} / \mathrm{s}$ at $Z=10^{-6} \mathrm{~m}$ [Frequency ranges considered: $0 \leq \omega \leq 0.78 \mathrm{rad} / \mathrm{s}$ and $\omega>0.78 \mathrm{rad} / \mathrm{s}]$. 


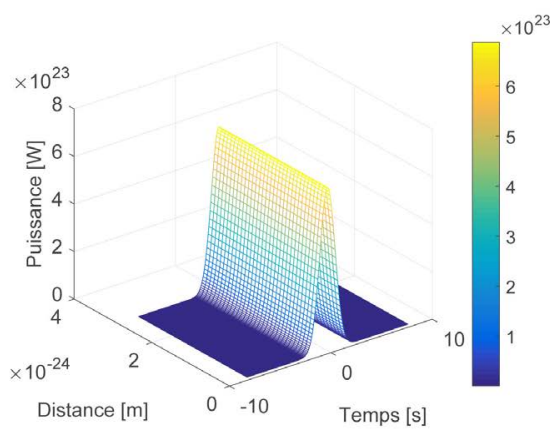

(a)

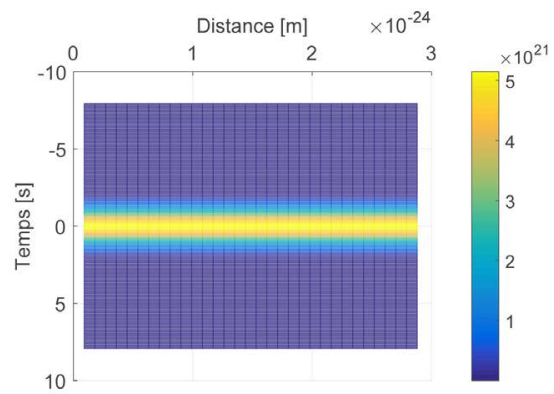

(c)

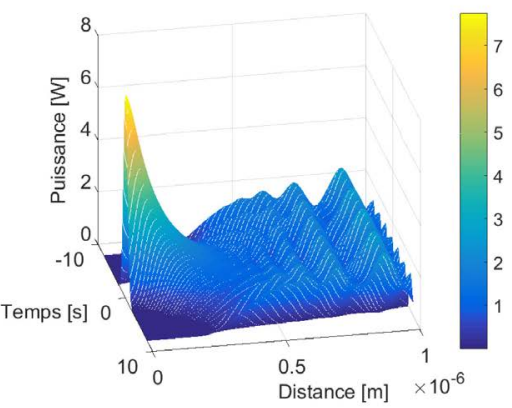

(b)

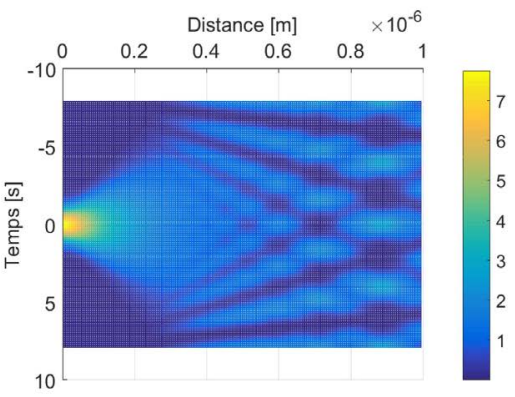

(d)

Figure 9. First case of left-handed behavior. Soliton light pulse and chaotic wave's field for $C_{r}=1$. Full equations: (a) $\omega=0.25 \mathrm{rad} / \mathrm{s}$; (b) $\omega=25 \mathrm{Mrad} / \mathrm{s}$. $2 \mathrm{D}$ Full equations: (c) $\omega=0.85 \mathrm{rad} / \mathrm{s}$; (d) $\omega=25 \mathrm{Mrad} / \mathrm{s}$. (a) and (c) at $Z=3 \times 10^{-24} \mathrm{~m}$; (b) and (d) at $Z=10^{-6} \mathrm{~m} \quad$ [Frequency ranges considered: $0 \leq \omega \leq 0.78 \mathrm{rad} / \mathrm{s}$ and $\omega>0.78 \mathrm{rad} / \mathrm{s}$ ].

The propagation is not favorable at high frequencies for left-handed behavior of the line. More so, the behavior of the line has changed. When right-handed behavior occurs the soliton light pulse propagates at high frequencies and it is destroyed at low frequencies. However, when left-handed behavior arrives the soliton light pulse propagates very well at low frequencies and it is totally destroyed at high frequencies. Hence, all these results suggest that the soliton light pulse propagates in opposite sense when the line changes its behavior from right-handed to left-handed. This result allows us to rename this transmission line as chameleon transmission line since its behavior changes without modify its physical aspect. This information is identical to that recently in [30].

\subsection{Impact of $C_{r}$ on Tree Structures}

We consider the case of right-handed propagation where $C_{r}=0$ at low frequencies. So, we obtain the tree structure depicted in Figure $6(\mathrm{c})$. This tree structure corresponds to rogue events signature where Akhmediev-Peregrine waves and Akhmediev waves trains appeared. At low frequencies, the rogue events which appear on the rogue signature depicted in Figure 6(c) are completely cancelled when $C_{r}=0.3$ as seen in Figure 7(b).

\subsubsection{Modified Chaotic Waves Field}

This figure corresponds to the signature of the stable soliton light pulse. So, the 
transmission line acts as bandpass filter at low frequencies. At high frequencies, when $C_{r}=1$ the left-handed behavior occurs and the rogue events signature is restored as seen in Figure 9(d). It is clearly observed that the number of big black points was increased from three to four as seen in Figure 6(c) and Figure 9(d). This result suggests that the radiations have increased in the system and they have provoked the multiplication of rogue events when $\omega=25 \mathrm{Mrad} / \mathrm{s}$. If the frequency is maintained and $C_{r}$ increases from 1 to 1.5 the number of black big points decreases from four to three as depicted in Figure $9(\mathrm{~d})$ and Figure 10(a). This situation denotes that the radiations have decreased in the system. So, the number of Akhmediev waves trains have decreased. If $C_{r}=10$ the radiations significantly increase leading to a strong perturbed chaotic waves field depicted in Figure 10(b). Besides, this perturbed system exhibits a strange tree structure illustrated in Figure 10(c). The precedent chaotic waves has changed into a strong perturbed chaotic waves field. The new field exhibits three Akhmediev-Peregrine rogue waves corresponding to the most colored points as seen in Figure 10(c). There are also some Peregrine waves represented by the least colored points. Otherwise, some Akhmediev waves trains are also represented.

\subsubsection{Modified Tree Structure}

In addition, when we pass from right-handed behavior $\left(C_{r}=0\right)$ to left-handed one $\left(C_{r}=1\right)$ the peak power decreases from $2.5 \times 10^{26} \mathrm{~W}$ to $7 \mathrm{~W}$ as seen in

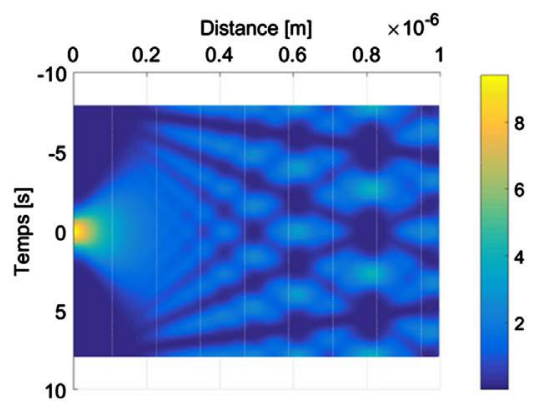

(a)

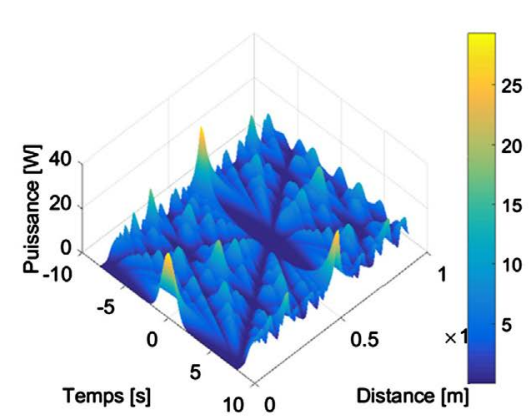

(b)

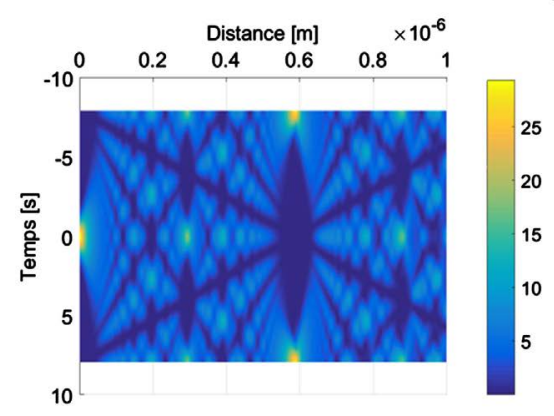

(c)

Figure 10. Influence of $C_{r}$ parameter on rogue events signature at $Z=10^{-6} \mathrm{~m}$ when $\omega=25 \mathrm{Mrad} / \mathrm{s}$. 2D Full equations: (a) $C_{r}=1.5$ and (c) $C_{r}=10$; (b) Full equation 
$C_{r}=10$.

Figure 6(c) and Figure 9(d). This brutal decrease of pulse peak power is not only due to the increase of the distance, but also due to a strange phenomenon. This strange phenomenon responsible to the strong decrease of peak power is called absorption. This phenomenon is normal because absorption is always observed in left-handed materials [45]. Besides, it has been demonstrated that in a realistic metamaterial system, absorption is unavoidable [45] [46] [47] [48]. In fact, some researchers such as Popov and Shalaev [45] sustain that absorption can be counteracted by optical amplification. Moreover, for left-handed behavior when $C_{r}$ increases from 1 to 10 the peak power also increases from $7 \mathrm{~W}$ to 25 $\mathrm{W}$ as depicted in Figure 9(d) and Figure 10(c). It appears that the dimensionless capacitor $C_{r}$ plays a crucial role in this transmission line. First, it modifies the aspect of rogue events signature. Second, it induces the multiplication of rogue events. Three, it introduces right- or left-handed behavior on the transmission line. Four, it modifies the pulse peak power. Five, it provokes the inversion of sense of waves propagation on the line. Six, it transforms the line into a bandpass filter at low frequencies.

\subsection{Influence of Frequency on Soliton Peak Power}

Moreover, when the dimensionless capacitor is neglected $\left(C_{r}=0\right)$ at right-handed behavior, the frequency significantly increases from $0.25 \mathrm{rad} / \mathrm{s}$ to $5 \mathrm{Mrad} / \mathrm{s}$. Then, the soliton peak power considerably decreases from $2.5 \times 10^{26} \mathrm{~W}$ to $14 \times 10^{20} \mathrm{~W}$. This situation is clearly illustrated in Figure 6(c) and Figure 9(d). So, an increase of frequency implies a decrease of pulse peak power in right-handed behavior of the line. However, if the right-handed propagation is maintained ( $C_{r}=0.3$ ), the frequency increases from $0.15 \mathrm{rad} / \mathrm{s}$ to $0.15 \mathrm{Mrad} / \mathrm{s}$. Moreover, the soliton peak power also decreases from $10 \times 10^{26} \mathrm{~W}$ to $5 \times 10^{3} \mathrm{~W}$ as depicted in Figure 7 (a) and Figure 7(b). Hence, this behavior of frequency is similar to that above mentioned. If the left-handed behavior $\left(C_{r}=1\right)$ occurs the frequency increases from $\omega=0.25 \mathrm{rad} / \mathrm{s}$ to $\omega=25 \mathrm{Mrad} / \mathrm{s}$. Consequently, the soliton peak power significantly decreases from $6 \times 10^{23} \mathrm{~W}$ to $7 \mathrm{~W}$ as depicted in Figure 9 (a) and Figure 9(b). It clearly appears that the influence of frequency on peak power strongly dependant on the value of dimensionless capacitor $C_{r}$ in the case of left-handed behavior.

\section{Conclusion}

In summary, we have studied an electromagnetic wave propagation when second-order dispersion and cubic-nonlinearity effects come into play. Those effects interact in a modulable electrical transmission line in order to build up dark or bright soliton. Thereafter, the manifestations exhibited for both right-handed and left-handed behaviors have been compared. Thus, collective coordinates technique of investigation has been used in order to study internal and external behaviors of electromagnetic light pulse. This investigation has been done at 
specific frequency ranges known as $0<\omega \leq 0.78 \mathrm{rad} / \mathrm{s}$ and $\omega>0.78 \mathrm{rad} / \mathrm{s}$. The numerical analysis leads to the following numerical outcomes. Firstly, we have verified that the line supports dark and bright soliton solutions. Thereafter, we also have verified that the line can exhibit chameleon's behavior. Indeed, we have shown that for right-handed behavior $\left(C_{r}=0\right)$, we obtain a chaotic waves field containing Akhmediev-Peregrine freak wave and Akhmediev freak waves trains, at low frequencies. However, this situation has been improved at high frequencies where a stable soliton has been obtained. Secondly, the second case of right-handed behavior $\left(C_{r}=0.3\right)$ has shown that the line acts as a bandpass filter at low frequencies and has maintained the robustness of the soliton at high frequencies. Thirdly, the left-handed behavior $\left(C_{r}=1\right)$ has restored the chaotic waves field at high frequencies and a stable soliton at low frequencies. Fourthly, we have presented the key role played by the dimensionless capacitor $C_{r}$ on the line. One, it has modified the aspect of rogue events signature and has induced the multiplication of rogue events. Two, it has introduced right- or left-handed behavior on the transmission line. Three, it also has modified the pulse peak power and has provoked the inversion of sense of waves propagation on the line.

\section{Conflicts of Interest}

The authors declare no conflicts of interest regarding the publication of this paper.

\section{References}

[1] Veselago, V.G. (1968) The Electrodynamics of Substances with Simultaneously Negative Values of $\varepsilon$ and $\mu$. Soviet Physics Uspekhi, 10, 509-514. https://doi.org/10.1070/PU1968v010n04ABEH003699

[2] Tütüncü, B., Torpi, H. and Urul, B. (2018) A Comparative Study on Different Types of Metamaterials for Enhancement of Microstrip Patch Antenna Directivity at the Ku-Band (12 GHz). Turkish Journal of Electrical Engineering \& Computer Sciences, 26, 1171-1179. https://doi.org/10.3906/elk-1711-50

[3] Caloz, C., Okabe, H., Iwai, T. and Itoh, T. (2002) Transmission Line Approach of Left-Handed (LH) Materials. USNCIURSI National Radio Science Meeting, San Antonio, June 2002, 39.

[4] Kozyrev, A.B. and Weide, D.W.V. (2008) Nonlinear Left-Handed Transmission Line Metamaterials. Journal of Physics D: Applied Physics, 41, 173001-173010. https://doi.org/10.1088/0022-3727/41/17/173001

[5] Lai, A., Caloz, C. and Itoh, T. (2004) Composite Right/Left-Handed Transmission Line Metamaterials. IEEE Microwave Magazine, 5, 34-50. https://doi.org/10.1109/MMW.2004.1337766

[6] Kharif, C., Pelinovsky, E. and Slunyaev, A. (2009) Rogue Waves in the Ocean. Springer, New York.

[7] Osborne, A.R. (2010) Nonlinear Ocean Waves and the Inverse Scattering Transform. Academic Press, Amsterdam.

[8] Solli, D.R., Ropers, C., Koonath, P. and Jalali, B. (2007) Optical Rogue Waves. Nature, 450, 1054-1057.https://doi.org/10.1038/nature06402 
https://www.nature.com/articles/nature06402

[9] Lecaplain, C., Grelu, P.H., Soto-Crespo, J.M. and Akhmediev, N. (2012) Dissipative Rogue Waves Generated by Chaotic Pulse Bunching in a Mode-Locked Laser. Physical Review Letters, 108, Article ID: 233901. https://doi.org/10.1103/PhysRevLett.108.233901

[10] Chabchoub, A., Hoffmann, N. and Akhmediev, N. (2011) Rogue Wave Observation in a Water Wave Tank. Physical Review Letters, 106, Article ID: 204502. https://doi.org/10.1103/PhysRevLett.106.204502

[11] Bailung, H., Sharma, S.K. and Nakamura, Y. (2011) Observation of Peregrine Solitons in a Multicomponent Plasma with Negative Ions. Physical Review Letters, 107, Article ID: 255005. https://doi.org/10.1103/PhysRevLett.107.255005

[12] Peregrine, D. (1983) Water Waves, Nonlinear Schrödinger Equations and Their Solutions. Journal of the Australian Mathematical Society Series, 25, 16-43. https://doi.org/10.1017/S0334270000003891

[13] Kuznetsov, E.A. (1977) Solitons in a Parametrically Unstable Plasma. Soviet Physics-Doklady, 22, 575-577.

[14] Ma, Y.C. (1979) The Perturbed Plane-Wave Solutions of the Cubic Schrödinger Equation. Studies in Applied Mathematics, 60, 43-58. https://doi.org/10.1002/sapm197960143

[15] Akhmediev, N., Eleonskii, V. and Kulagin, N. (1987) Exact First-Order Solutions of the Nonlinear Schrödinger Equation. Theoretical and Mathematical Physics, 72, 809-818. https://doi.org/10.1007/BF01017105

[16] Zakharov, V. and Ostrovsky, L. (2009) Modulation Instability: The Beginning. Physica D: Nonlinear Phenomena, 238, 540-548. https://doi.org/10.1016/j.physd.2008.12.002

[17] Kelleher, E.J.R., Travers, J.C., Popov, S.V. and Taylor, J.R. (2012) Role of Pump Coherence in the Evolution of Continuous-Wave Supercontinuum Generation Initiated by Modulation Instability. Journal of the Optical Society of America B, 29, 502-512. https://doi.org/10.1364/JOSAB.29.000502

[18] Dudley, J.M., Genty, G., Dias, F., Kibler, B. and Akhmediev, N. (2009) Modulation Instability, Akhmediev Breathers and Continuous Wave Supercontinuum Generation. Optics Express, 17, 21497-21508. https://doi.org/10.1364/OE.17.021497

[19] Dysthe, K.B. and Trulsen, K. (1999) Note on Breather Type Solutions of the NLS as Models for Freak-Waves. Physica Scripta, 1999, Article No. T82. https://doi.org/10.1238/Physica.Topical.082a00048

[20] Bespalov, I. and Talanov, V.I. (1966) Filamentary Structure of Light Beams in Nonlinear Liquids, Journal of Experimental and Theoretical Physics Letters, 3, 307-309.

[21] Hasegawa, A. and Kodama, Y. (1995) Solitons in Optical Communications. Clarendon Press, Oxford.

[22] Benjamin, T.B. and Feir, J.E.J. (1967) The Disintegration of Wavetrains on Deep Water. Fluid Mechanics, 27, 417-430. https://doi.org/10.1017/S002211206700045X

[23] Marqui?, P., Bilbault, J.M. and Remoissenet, M. (1995) Observation of Nonlinear Localized Modes in an Electrical Lattice. Physical Review E, 51, 6127. https://doi.org/10.1103/PhysRevE.51.6127

[24] Ndi Nnanga, B.M., Gouadjio Dontsop, P.Y., Onana Essama, B.G., Shabat, M., Yemele, D. and Atangana, J. (2020) Tree-Like Structures and Freak Waves Generation Induced by Quintic. Nonlinearity and Cubic. Raman Effect in a Nonlinear Metamaterial. Optical and Quantum Electronics, 52, Article No. 356. 
https://doi.org/10.1007/s11082-020-02469-4

[25] Charalampidis, E.G., Cuevas-Maraver, J., Frantzeskakis, D.J. and Kevrekidis, P.G. (2018) Rogue Waves in Ultracold Bosonic Seas. Romanian Reports in Physics, 70, Article No. 504.

[26] Bertola, M. and Tovbis, A. (2013) Universality for the Focusing Nonlinear Schr ödinger Equation at the Gradient Catastrophe Point: Rational Breathers and Poles of the Tritronquée Solution to Painlevé I. Comm. Communications on Pure and Applied Mathematics, 66, 678-752. https://doi.org/10.1002/cpa.21445

[27] Bayindir, C. (2016) Rogue Waves of the Kundu-Eckhaus Equation in a Chaotic Wave Field. Physical Review E, 93, Article No. 062215.

https://doi.org/10.1103/PhysRevE.93.032201

[28] Dudley, J.M., Dias, F., Erkintalo, M. and Genty, G. (2014) Instabilities, Breathers and Rogue Waves in Optics. Nature Photonics, 8, 755-764. https://doi.org/10.1038/nphoton.2014.220

[29] Fukushima, K., Wadati, M. and Narahara, Y. (1980) Envelope Soliton in a New Nonlinear Transmission Line. Journal of the Physical Society of Japan, 49, 1593-1597. https://doi.org/10.1143/JPSJ.49.1593

[30] Togueu Motcheyo, A.B., Tchinang Tchameu, J.D., Fewo, S.I., Tchawoua, C. and Kofane, T.C. (2017) Chameleon's Behavior of Modulable Nonlinear Electrical Transmission Line. Communications in Nonlinear Science and Numerical Simulation, 53, 22-30.https://doi.org/10.1016/j.cnsns.2017.04.031

[31] Tchofo Dinda, P., Nakkeeran, K. and Labruyere, A. (2002) Suppression of Soliton Self-Frequency Shift by Upshifted Filtering. Optics Letters, 27, 382-384. https://doi.org/10.1364/OL.27.000382

[32] Hirota, R. and Suzuki, K. (1970) Studies on Lattice Solitons by Using Electrical Networks. Journal of the Physical Society of Japan, 28, 1366-1367.

https://doi.org/10.1143/JPSJ.28.1366

[33] Hirota, R. and Suzuki, K. (1973) Theoretical and Experimental Studies of Lattice Solitons in Nonlinear Lumped Networks. Proceedings of the IEEE, 61, 1483-1491. https://doi.org/10.1109/PROC.1973.9297

[34] Agrawal, G.P. (1995) Nonlinear Fiber Optics. 2nd Edition, Academic Press, San Diego.

[35] Onana Essama, B.G., Atangana, J., Mokhtari, B., Cherkaoui Eddeqaqi, N. and Kofane, T.C. (2013) Theoretical Model for Electromagnetic Wave Propagation in Negative Index Material Induced by Cubic-Quintic Nonlinearities and Third-Order Dispersion Effects. Optical and Quantum Electronics, 46, 911-924. https://doi.org/10.1007/s11082-013-9804-Z

[36] Onana Essama, B.G., Atangana, J., Biya Motto, F., Mokhtari, B., Cherkaoui Eddeqaqi, N. and Kofane, T.C. (2014) Rogue Waves Generation in a Left-Handed Nonlinear Transmission Line with Series Varactor Diodes. Journal of Modern Optics, 61, 1002-1008. https://doi.org/10.1080/09500340.2014.917728

[37] Onana Essama, B.G., Atangana, J., Biya Motto, F., Mokhtari, B., Cherkaoui Eddeqaqi, N. and Kofane, T.C. (2014) Optical Rogue Waves Generation in a Nonlinear Metamaterial. Optics Communications, 331, 334-347.

https://doi.org/10.1016/j.optcom.2014.06.039

[38] Onana Essama, B.G., Atangana, J., Biya Motto, F., Mokhtari, B., Cherkaoui Eddeqaqi, N. and Kofane, T.C. (2014) Rogue Wave Trains Generation in a Metamaterial Induced by Cubic-Quintic-Nonlinearities and Second-Order Dispersion. Physical 
Review E, 90, Article ID: 032911.

https://doi.org/10.1103/PhysRevE.90.032911

[39] Atangana, J., Onana Essama, B.G., Mokhtari, B. and Kofane, T.C. (2013) Cubic-Quintic Saturable Nonlinearity Effects on a Light Pulse Strongly Distorted by the Fourth-Order Dispersion. Journal of Modern Optics, 60, 292-300. https://doi.org/10.1080/09500340.2013.772665

[40] Tchofo Dinda, P., Moubissi, A.B. and Nakkeeran, K. (2001) A Collective Variable Approach for Dispersion-Managed Solitons. Journal of Physics A: Mathematical and General, 34, L103. https://doi.org/10.1088/0305-4470/34/10/104

[41] Atangana, J., Onana Essama, B.G., Biya Motto, F., Mokhtari, B., Cherkaoui Eddeqaqi, N. and Kofane, T.C. (2014) Kuznetsov-Ma Waves Train Generation in a Left-Handed Material. Journal of Modern Optics, 62, 392-402.

https://doi.org/10.1080/09500340.2014.986234

[42] Atangana, J., Ndi Nnanga, B.M., Onana Essama, B.G., Mokthari, B., Cherkaoui Eddeqaqi, N. and Kofane, T.C. (2015) Efficient Method of Calculation of Raman Soliton Self-Frequency Shift in Nonlinear Optical Media. Optics Communications, 339, 194-208.

[43] Gouadjio Dontsop, P.Y., Onana Essama, B.G., Dongo, J.M., Mbou Dedzo, M., Atangana, J., Yemele, D. and Kofane, T.C. (2016) Akhmediev-Peregrine Rogue Waves Generation in a Composite Right/Left-Handed Transmission Line. Optical and Quantum Electronics, 48, Article No. 59. https://doi.org/10.1007/s11082-015-0333-9

[44] Vitanov, N.K., Chabchoub, A. and Hoffmann, N. (2013) Deep-Water Waves: On the Nonlinear Schrödinger Equation and Its Solutions. Journal of Theoretical and Applied Mechanics, 43, 43-54.

[45] Popov, A.K. and Shalaev, V.M. (2006) Compensating Losses in Negative-Index Metamaterials by Optical Parametric Amplification. Optics Letters, 31, 2169-2171. https://doi.org/10.1364/OL.31.002169

[46] Litchinitser, N.M. and Shalaev, V.M. (2009) Loss as a Route to Transparency. Nature Photonics, 3, 75-79. https://doi.org/10.1038/nphoton.2008.280

[47] Onana Essama, B.G., Ndjakomo Essiane, S., Biya-Motto, F., Nnanga, B.M.N., Shabat, M. and Atangana, J. (2020) Dynamical Evolution of Sasa-Satsuma Rogue Waves, Breather Solutions, and New Special Wave Phenomena in a Nonlinear Metamaterial. Physica Status Solidi (B), Online Version. https://doi.org/10.1002/pssb.202000316

[48] Onana Essama, B.G., Ndjakomo Essiane, S., Biya-Motto, F., Shabat, M. andAtangana, J. (2020) Triangular Rogue Waves and Multi-Wave Trains Generation in a Chameleon Electrical Transmission Line. American Journal of Optics and Photonics, 8, 61-73. 